\title{
Clinical Significance of Cystatin C, Creatinine, and Blood Urea in Lupus Nephritis Patients with Systemic Lupus Erythematosus: A South Indian Case-Control Study
}

Bhanuja Rani ${ }^{1}$, Mohammed Suleman ${ }^{2}$, CV Sarada $^{3}$, Madhavi Latha Manolla ${ }^{4}$, T Kinnera ${ }^{5}$, Lavanya Maccha ${ }^{6}$, N Gayatri ${ }^{7}$, Sujatha Rani ${ }^{8}$, Dinesh Yadav ${ }^{9}$, Gokul Vinod Kumar ${ }^{10}$

\begin{abstract}
Lupus nephritis (LN) develops as a result of immunological abnormalities. The pathogenesis of LN is a complex process, involving the deposition of autoantibodies in the glomerulus. The glomerular filtration rate (GFR) is widely accepted as the best overall measure of kidney function, enabling a statement of the complex functions of the kidney in a single numeric expression. The endogenous marker of GFR commonly employed is creatinine, but it does not complete the requirements of an ideal marker because apart from being subjected to tubular secretion it is also influenced by the muscle mass and gender of the patient. Cystatin C is a protein produced by all nucleated cells in the body, freely filtered by glomerulus; neither returned to the bloodstream nor secreted by the renal tubules and also is not influenced by gender or muscle mass. The above features make it a better marker of renal function than creatinine. The medical therapy for LN depends on the severity of the disease. Finding reliable biomarkers for LN will help to evaluate disease activity, identify patients at risk for kidney damage, and facilitate early diagnosis and intervention to improve favorable outcomes.

Keywords: Creatinine, Cystatin C, Glomerular filtration rate, Lupus, Lupus nephritis, Systemic lupus erythematosus.

Indian Journal of Medical Biochemistry (2021): 10.5005/jp-journals-10054-0172
\end{abstract}

\section{INTRODUCTION}

Lupus nephritis $(\mathrm{LN})$ is a complication affecting the prognosis of patients with systemic lupus erythematosus (SLE), a representative type III allergy caused by immune complexes (ICs). Systemic lupus erythematosus is the most characteristic autoimmune disorder that can lead to tissue damage in many organs, including the kidney. Generally, $74 \%$ of lupus patients develop clinically relevant nephritis at some time during the course of their illness. ${ }^{1}$ Lupus nephritis develops as a result of immunological abnormalities. The pathogenesis of $L N$ is a complex process, involving the deposition of autoantibodies in the glomerulus. Lupus nephritis is the disease of the kidneys due to the deposition of autoantibodies that cause inflammation. About $30-50 \%$ of lupus patients will develop LN within the first 6 months to 3 years of being diagnosed with SLE. ${ }^{2}$

The glomerular filtration rate (GFR) is widely accepted as the best overall measure of kidney function, enabling a statement of the complex functions of the kidney in a single numeric expression. ${ }^{3} \mathrm{An}$ ideal marker for GFR should be constantly produced, freely filtered, not reabsorbed or secreted by the renal tubules, and metabolized or eliminated by extrarenal mechanisms. The endogenous marker of GFR commonly employed is creatinine, but it does not complete the requirements of an ideal marker because apart from being subjected to tubular secretion it is also influenced by the muscle mass and gender of the patient. ${ }^{4}$

Cystatin $C$ is a protein produced by all nucleated cells in the body, freely filtered by glomerulus; neither returned to the bloodstream nor secreted by the renal tubules and also is not influenced by gender or muscle mass. ${ }^{5}$ The above features make it a better marker of renal function than creatinine. ${ }^{6}$ The medical therapy for LN depends on the severity of the disease. Finding reliable biomarkers for LN will help to evaluate disease activity, identify patients at risk
1-3,5,6 Department of Biochemistry, Gandhi Medical College and Hospital, Secundrabad, Telangana, India

${ }^{4,9}$ Multidisciplinary Research Unit, Gandhi Medical College and Hospital, Secundrabad, Telangana, India

${ }^{7}$ Department of Social and Preventive Medicine, RVM Medical College, Mulug, Siddipet, Telangana, India

${ }^{8}$ Department of Biochemistry, Osmania Medical College, Hyderabad, Telangana, India

${ }^{10}$ Department of Social and Preventive Medicine, Gandhi Medical College and Hospital, Secundrabad, Telangana, India

Corresponding Author: Bhanuja Rani, Department of Biochemistry, Gandhi Medical College and Hospital, Secundrabad, Telangana, India, Phone: +91 9848043837, e-mail: mdrugmc19@gmail.com

How to cite this article: Rani B, Suleman M, Sarada CV, et al. Clinical Significance of Cystatin C, Creatinine and Blood Urea in Lupus Nephritis Patients with Systemic Lupus Erythematosus: A South Indian Case-Control Study. Indian J Med Biochem 2021;25(1):9-13.

Source of support: Nil

Conflict of interest: None

for kidney damage, and facilitate early diagnosis and intervention to improve favorable outcomes. Therefore, this study is undertaken to assess the most reliable marker among the renal parameters, i.e., cystatin $C$, serum creatinine, blood urea, and proteinuria for diagnosing early-stage kidney damage in SLE patients.

\section{Materials and Methods}

This was a case-control (50 cases and 50 controls), prospective and observational study, including patients with SLE attending 
the autoimmune disease outpatient and inpatient attending to Dermatology, General medicine, Pediatrics are taken into this study and selected from over a period of one and a half year from March 2014 to October 2015. Blood samples of SLE patients are collected.

The single criteria for inclusion in the study were the presence of diagnosed SLE according to the American College of Rheumatology 1997 criteria. $^{7}$ The exclusion criteria included the presence of any kidney disease with other etiology like diabetes, pregnant women, and thyroid disease.

The present study involved 100 subjects, of whom 50 were SLE cases attended outpatient and inpatient Departments of Gandhi Hospital who fulfilled the inclusion criteria and the other 50 were age and sex-matched controls. The present study was undertaken to evaluate the cystatin C, creatinine, blood urea, and proteinuria levels in SLE patients. In the present study, serum cystatin C was measured by the Nephelometric immunoassay, serum creatinine by the enzymatic method in Mespa xl 240, blood urea by the urease single-step method in auto analyzer ERBA 640, and proteinuria by the Sulfo-salicylic acid method. The results were tabulated in the master chart and statistically analyzed using Microsoft Excel 2007 and SPSS version 15.0.

This study was approved by the Institutional Ethics Committee, Gandhi medical college (Letter No: IJMB0172).

\section{Assessed Variables}

Different parameters were assessed from all the patients included in the study. Serum cystatin C level was determined by nephelometry (reference values in individuals up to 50 years: $0.55-1.15 \mathrm{mg} /$ individuals above 50 years: $0.63-1.44 \mathrm{mg} / \mathrm{L}$ ) creatinine by modified Jaffe's method (reference value: $0.5-1.1 \mathrm{mg} / \mathrm{dL}$ ), urea in blood estimated by urease method ${ }^{7}$ at Clinical Chemistry Laboratory, Gandhi Hospital attached to Gandhi Medical College, Secunderabad were processed. To assess a possible influence on serum.

The present study involved 100 subjects, of whom 50 were SLE cases attended outpatient and inpatient Departments of Gandhi Hospital who fulfilled the inclusion criteria and the other 50 were age and sex-matched controls. The present study was undertaken to evaluate the cystatin C, creatinine, blood urea, and proteinuria levels in SLE patients. In the present study, serum cystatin $C$ was measured by the Nephelometric immunoassay, serum creatinine by the enzymatic method in Mespa xl 240, blood urea by the urease single-step method in auto analyzer ERBA 640, and proteinuria by the Sulfo-salicylic acid method. The results were tabulated in the master chart and statistically analyzed using Microsoft Excel 2007 and SPSS version 15.0 .

\section{Statistical Analysis}

Results on continuous measurements are presented on mean and SD and results on categorical measurements are presented in number (\%). Significance is assessed at a $5 \%$ level of significance. Independent Student's $t$-test (two-tailed, independent) was used to find the significance of study parameters on a continuous scale between two groups (intergroup analysis) on metric parameters. Chi-square/Fisher's exact test was used to find the significance of study parameters on a categorical scale between two groups.

\section{Results}

\section{Gender Distribution among Cases and Controls}

In the present study, female predominance was observed with 47 were females $94 \%$ and 3 were males with $6 \%$ in both cases and controls.

\section{Age Distribution}

The distribution of the study subjects according to age is given in Table 1 and graphically represented. The cases and controls are divided into four groups ( $<20,21-25,26-30$, and $>30$ years). Maximum numbers of cases are in the age-group of $20(36 \%)$ (Fig. 1).

\section{Distribution of Serum Cystatin C}

A comparison of serum cystatin $C$ between cases and controls is shown in Table 2. All the controls were with normal cystatin C levels. Among cases, three (6\%) were with normal cystatin C levels. The majority among cases, 47 (94\%) were with elevated cystatin C levels. None of the controls were with elevated cystatin C levels (Table 3 ).

\section{Comparison of Serum Creatinine between Cases and Controls}

A comparison of serum creatinine between cases and controls is shown in Figure 1. All the controls were with normal creatinine levels. Among cases, the majority 43 (86\%) were with normal creatinine levels followed by 7 (14\%) were with elevated creatinine levels.

\section{Distribution of Blood Urea ( $\mathrm{mg} / \mathrm{dL}$ ) Level among the Two Groups (Table 1)}

The distribution of blood urea among cases and controls is given in Figure 1. All the controls were within normal blood urea levels. Among cases, the majority 43 (86\%) were within the normal range followed by 7 (14\%) were with elevated levels (Tables 4 and 5). Among cases, the mean values for cystatin $C$ and creatinine were 1.502 and 0.906 , respectively, with SD 0.40323 and 0.18296 and the mean difference is statistically significant $(p<0.01)$. There is a positive correlation between cystatin $C$ and creatinine levels among cases. The overall correlation coefficient for the reciprocal of serum Cys $\mathrm{C} r=0.235 ; 95 \%$ confidence interval $(\mathrm{Cl})$, was superior to that of the reciprocal of serum creatinine; $95 \% \mathrm{Cl}, p<0.0000001$ (Table 2).

Among cases, mean values for cystatin $C$ and blood urea were 1.502 and 28.18, respectively, with SD 0.40323 and 13.057 and the mean difference is statistically significant $(p<0.01)$. There is a positive correlation between cystatin $C$ and blood urea levels among cases. The overall correlation coefficient for the reciprocal of serum Cys $\mathrm{C} r=0.042 ; 95 \% \mathrm{Cl}$, was superior to that of the reciprocal of blood urea; $95 \% \mathrm{Cl}, p<0.0000001$ (Fig. 2). Receiver-operator characteristics (ROC) curve analysis was performed to find cystatin $C$ as a diagnostic marker and diagnostic statistics viz. sensitivity, specificity, PPV, NPV, and accuracy have been computed to find the correlation. Receiver-operator characteristics curve analysis for finding the diagnostic role of cystatin $C$, the area under the curve 0.958 with $88 \%$ sensitivity and $84 \%$ specificity with $p$ value 0.001 which is statistically significant (Fig. 3).

Table 1: Comparison of mean and SD of serum creatinine $(\mathrm{mg} / \mathrm{dL})$ between the two groups $(N=100)$

\begin{tabular}{llll}
\hline Group & Mean & $S D$ & $p$ value \\
\hline $\begin{array}{l}\text { Controls } \\
(n=50)\end{array}$ & 0.886 & 0.182958 & 0.08 \\
Cases $(n=50)$ & 0.906 & 0.234225 & \\
\hline
\end{tabular}

Mean \pm SD of serum creatinine is higher in cases than in controls and the mean difference is not statistically significant $(p>0.05)$ 

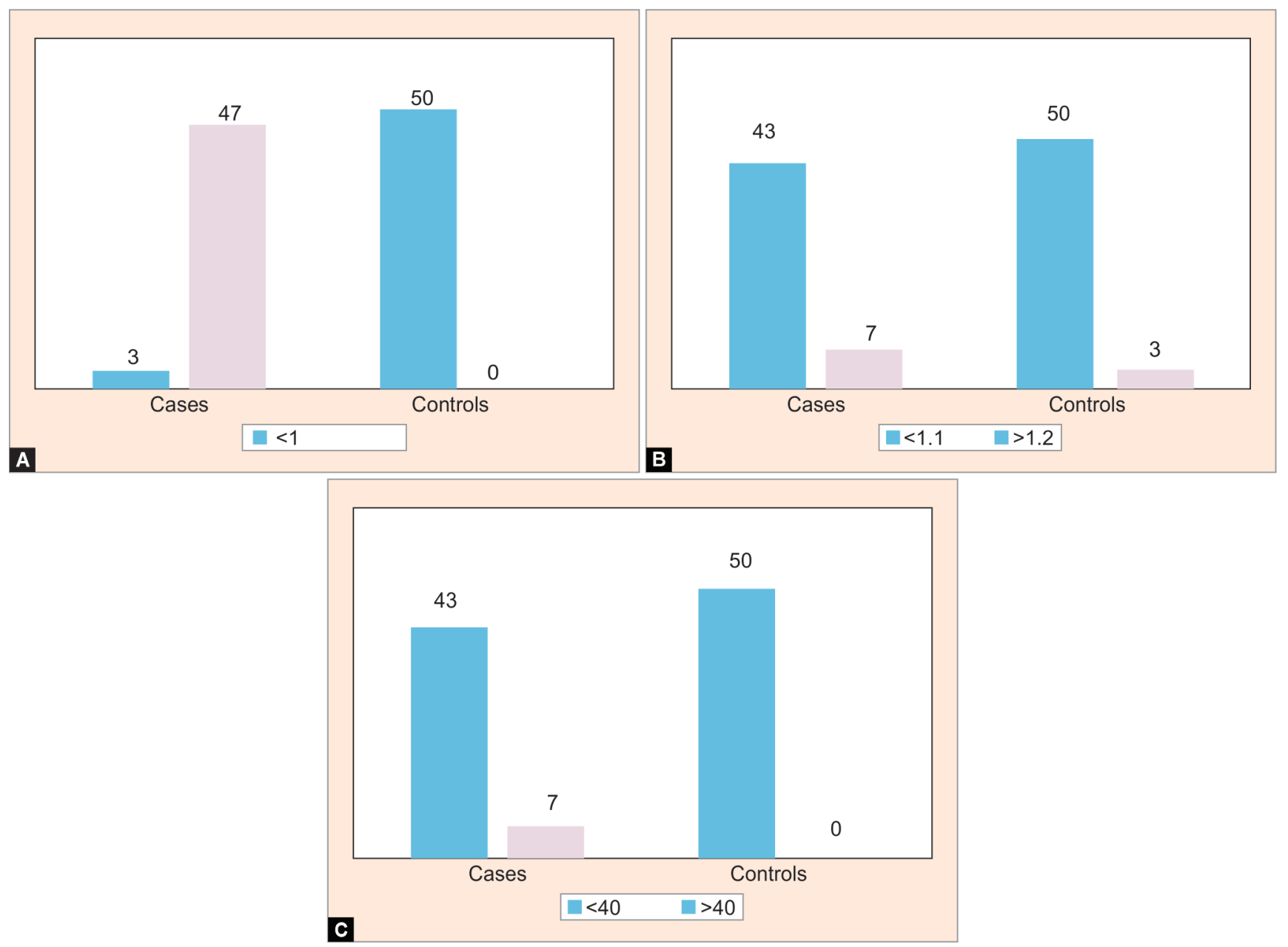

Figs $1 \mathrm{~A}$ to C: Distribution of cystatin C, creatinine, and blood urea levels among cases and controls

Table 2: Correlation between cystatin $C$ and serum creatinine among cases

\begin{tabular}{lllll}
\hline Methods & Mean & $S D$ & rvalue & $p$ value \\
\hline Cystatin C & 1.502 & 0.40323 & 0.235 & $<0.0000001$ \\
Creatinine & 0.906 & 0.18296 & & \\
\hline
\end{tabular}

Table 3: Comparison of mean \pm SD of cystatin $C$ between the two groups

\begin{tabular}{llll}
\hline Group & Mean & SD & $p$ value \\
\hline $\begin{array}{l}\text { Controls } \\
(n=50)\end{array}$ & 0.656 & 0.12803 & $<0.0001$ \\
Cases $(n=50)$ & 1.502 & 0.40323 & \\
\hline
\end{tabular}

Mean \pm SD of cystatin C between the two groups is statistically significant $(p<0.01)$

Table 4: Comparison of mean and SD of blood urea $(\mathrm{mg} / \mathrm{dL})$ between the two groups $(N=100)$

\begin{tabular}{llll}
\hline Group & Mean & $S D$ & $p$ value \\
\hline $\begin{array}{l}\text { Controls } \\
(n=50)\end{array}$ & 25.18 & 7.02931 & $<0.00002$ \\
Cases $(n=50)$ & 28.18 & 13.05669 & \\
\hline
\end{tabular}

Mean \pm SD of blood urea is higher in cases than in controls and the mean difference is statistically significant $(p<0.01)$

\section{Discussion}

Systemic lupus erythematosus is a prototype of systemic autoimmune diseases. A prevalence study in India carried out in a rural population near Delhi found a point prevalence of 3 per 100,000 populations. ${ }^{7}$ However, a fair number of cases of SLE are encountered in any large hospital in India. Despite its rarity, SLE has a considerable impact on the patient, her/his family, and the health services available.

The etiology is not known as yet and the pathogenesis is complex, involving immunological, genetic, hormonal, and environmental factors. Damage to tissues and cells results from pathogenic autoantibodies and ICs. ${ }^{8}$ Remissions and relapses characterize the disease. The clinical manifestations and their severity in individual patients may vary considerably and, therefore, the treatment strategy needs to be tailored accordingly.

In the present study, the median age of onset of SLE was 25 years, and the female:male ratio was 16:1. It is correlating with the above study.

Lupus nephritis is currently defined as the presence of more than +++ or $0.5 \mathrm{~g} / 24$ hours proteinuria or the presence of cellular casts of any type. ${ }^{9}$ Lupus nephritis occurs in about half of SLE patients range from 35 to $73 \%$ in India. It is rare for $L N$ to present with progressive renal insufficiency with repeatedly normal urine analysis. Also, nephritic range proteinuria is uncommon (app. 10\%) 
Table 5: Comparison of all renal parameters

\begin{tabular}{|c|c|c|c|c|c|}
\hline \multirow[b]{2}{*}{ Methods } & \multicolumn{2}{|c|}{ Cases } & \multicolumn{2}{|c|}{ Controls } & \multirow[b]{2}{*}{ pvalue } \\
\hline & Mean & $S D$ & Mean & $S D$ & \\
\hline Cystatin & 1.502 & 0.40323 & 0.656 & 0.12803 & $<0.0000001$ \\
\hline Creatinine & 0.906 & 0.18296 & 0.886 & 0.234225 & 0.08695 \\
\hline Urea & 28.18 & 13.057 & 25.18 & 7.021 & 0.00002839 \\
\hline
\end{tabular}
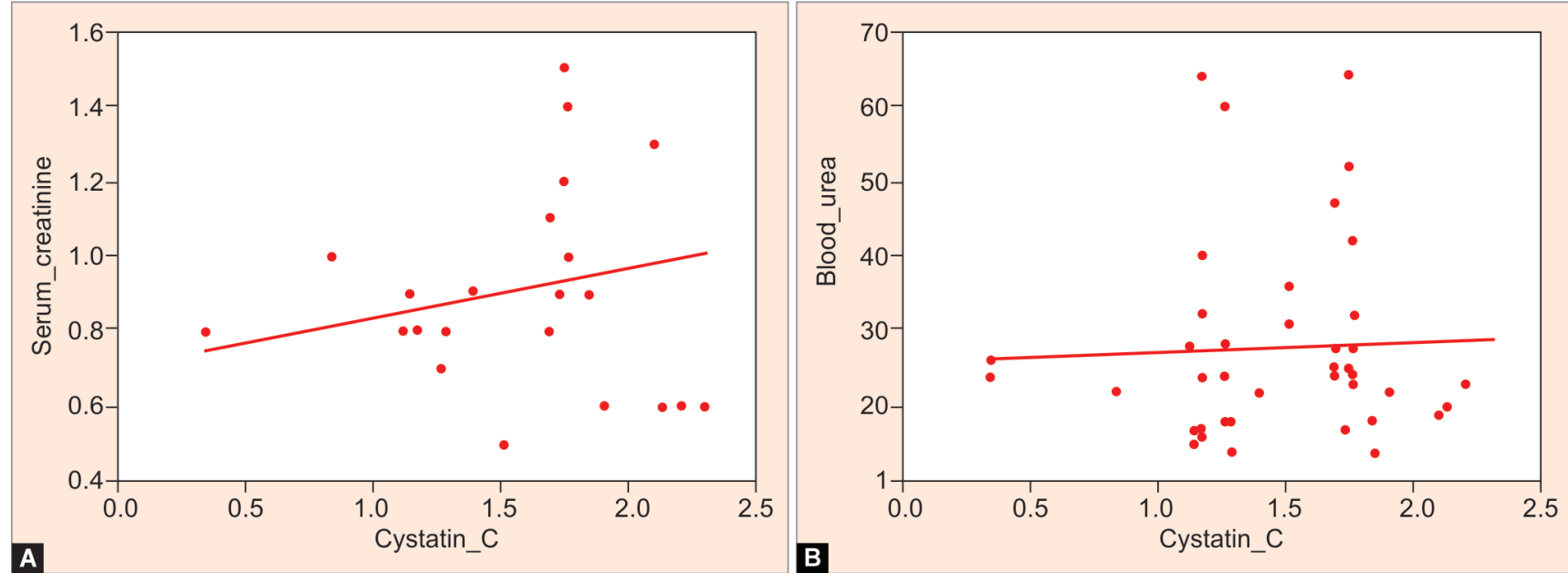

Figs $2 \mathrm{~A}$ and B: Correlation between cystatin, serum creatinine and blood urea

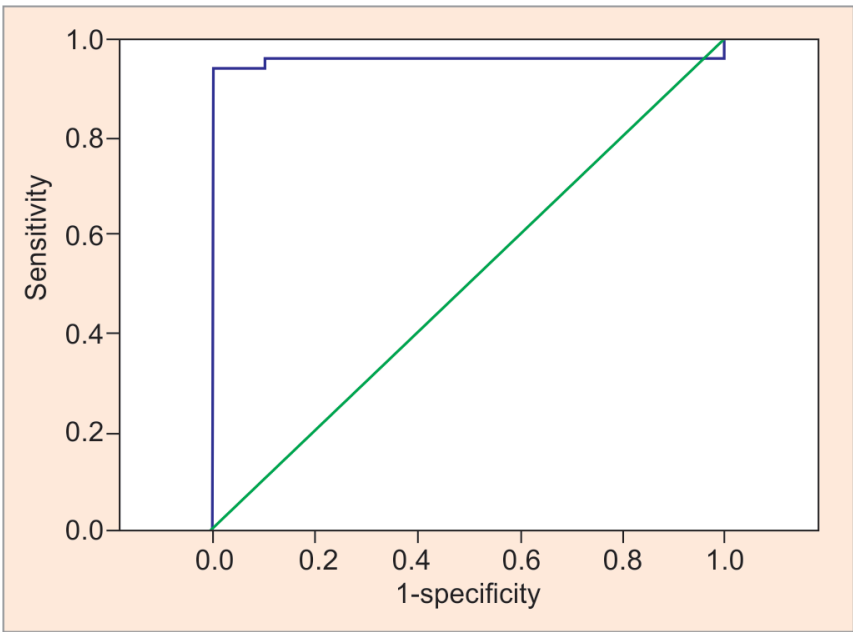

Fig. 3: Receiver operative curve for cystatin $C$ among cases and controls. ROC curve analysis for finding the diagnostic role of cystatin $C$, area under the curve 0.958 with $88 \%$ sensitivity and $84 \%$ specificity with $p$ value 0.001 which is statistically significant

in Indian lupus. Thus, a reliable urine examination is the single most important investigation for early diagnosis of $\mathrm{LN}$; the clinical examination is generally unhelpful. ${ }^{10}$ In the present study, none of the patients had proteinuria.

Lupus nephritis develops as a result of immunological abnormalities. The pathogenesis of LN is a complex process, involving the deposition of autoantibodies in the glomerulus, activation of complement and macrophages, cell proliferation. Production of extracellular matrix proteins, pro-inflammatory cytokines, and chemokine, cause tubular damage, tubular interstitial inflammation, and fibrosis, leading to renal dysfunction which increases the mortality rate of SLE patients, it is necessary to assess renal function in these patients very precisely. ${ }^{11}$ Various studies are suggesting that serum cystatin $C$ is sensitive than creatinine. Hence, it may help in the detection of renal dysfunction early in SLE patients. Early detection of renal damage in SLE patients can help in the administration of proper treatment and to take precautionary measures to prevent further decline of renal function and thereby preventing the development of LN.

Serum cystatin $C$ is superior to serum creatinine as a marker of kidney function. Serum cystatin $C$ has been proposed as a simple, accurate, and rapid endogenous marker of GFR in research and clinical practice. ${ }^{12}$ However, there are conflicting reports regarding the superiority of Cyst $C$ over serum creatinine, with many studies suggesting significant.

In the present study, the mean value for cystatin $C$ and creatinine were 1.502 and 0.906 with SD 0.43 and 0.18 , respectively, with $p$ value $<0.001$. There is a positive correlation between cystatin $\mathrm{C}$ and creatinine in cases. The overall correlation coefficient for the reciprocal of serum cystatin $\mathrm{Cr}=0.235,95 \% \mathrm{Cl}$ was superior to that of serum creatinine, $95 \% \mathrm{Cl}$ and $p<0.0000001$. Receiver-operator characteristics curve analysis for finding the diagnostic role of cystatin C area under the curve 0.958 with $88 \%$ sensitivity and $84 \%$ specificity with $p$ value 0.001 .

Martinez-Martinez et al. ${ }^{13}$ did a study in 60 consecutive patients with SLE in the order in which the blood sample was taken to determine levels of creatinine (creatinine standardized by the Roche enzymatic method) ${ }^{14}$ and cystatin C (immunonephelometric test particles). He found that mean \pm SD of cystatin C (1.16 \pm 1.0$)$, mean age was $(36.8 \pm 15.1)$, time since the onset of SLE, months $(85.3 \pm 84.60 .107)$ and mean of $\mathrm{S} \mathrm{Cr}, \mathrm{mg} / \mathrm{dL} 1.1 \pm 1.50 .778$. Finally, they concluded that cystatin $C$ is a better renal marker than serum creatinine. In this study, the mean \pm SD of creatinine level in cases 
was $0.91 \pm 0.23$. Mean \pm SD of creatinine level in controls was $0.89 \pm 0.18$, so there is no significance. Mean \pm SD of serum cystatin $C$ in cases is $1.5 \pm 0.4$ and in controls is $0.6 \pm 0.12$ with $p$ values $<0.0000001$ so, cystatin $C$ is a better marker.

In the present study, on 100 individuals where 50 are SLE cases, the results of their mean \pm SD age, years $26.82 \pm 10.1$, mean \pm SD cystatin $C$ is $1.5 \pm 0.4$, mean $\pm S D$ of $S C r$ is $0.9 \pm 0.23$. The correlation $(r)$ between cystatin $C$ and serum creatinine was 0.235 with $p$ value $<0.001$.

In the present study, cystatin $C$ concentration as a marker of renal function in patients with SLE where serum cystatin $C$, serum creatinine, and blood urea were measured and analyzed. Serum cystatin C concentrations good significance ( $p=0.0000001$ ), creatinine concentrations mean \pm SD: $0.90 \pm 0.23$ vs $0.88 \pm 0.18$ ( $p=0.089$ ) in cases and controls, so there was no significant difference in creatinine concentrations. Blood urea concentrations mean \pm SD: $28.18 \pm 13.06$ vs $25.18 \pm 7.03(p=0.00003)$, higher in cases than controls. Stating that serum cystatin $C$ and blood urea are higher in cases and significant. Receiver-operator characteristics revealed a differential diagnostic advantage of cystatin $C$ over creatinine and urea.

\section{Limitations of the Study}

- The study was conducted on a limited sample over a short duration of time. Large scale study over a long duration is required to observe the more significant changes in serum cystatin $C$ and serum creatinine levels.

- The study was conducted on SLE diagnosed by ARA Criteria, but not SLEDAI.

- Measurement of GFR would have helped in grading renal insufficiency, impaired renal function missed by serum creatinine was correctly analyzed by cystatin C.

\section{References}

1. Neidhart M, Pataki F, Michel BA, et al. CD45 isoforms expression on CD4+ and CD8+ peripheral blood T-lymphocytes is related to autoimmune processes and hematological manifestations in systemic lupus erythematosus. Schweiz Med Wochenschr 1996;126(45): 1922-1925.
2. Oates JC, Gilkeson GS. Mediators of injury in lupus nephritis. Curr Opin Rheumatol 2002;14(5):498-503. DOI: 10.1097/00002281-20020900000003.

3. Stevens LA, Levey AS. Measured GFR as a confirmatory test for estimated GFR. J Am Soc Nephrol 2009;20(11):2305-2313. DOI: 10.1681/ASN.2009020171.

4. Noble J, Jouve T, Malvezzi P, et al. Renal complications of liver diseases. Expert Rev Gastroenterol Hepatol 2018;12(11):1135-1142. DOI: 10.1080/17474124.2018.1530984.

5. Chang MY, Park Y, Ha JW, et al. Paraspinal lean muscle mass measurement using spine MRI as a predictor of adjacent segment disease after lumbar fusion: a propensity score-matched case-control analysis. JR Am J Roentgenol 2019;12:1-8. DOI: 10.2214/AJR.18.20441.

6. Pillemer SR, Fowler SE, Tilley BC, et al. Meaningful improvement criteria sets in a rheumatoid arthritis clinical trial. MIRA trial group. Minocycline in rheumatoid arthritis. Arthritis Rheum 1997;40(3):419425. DOI: 10.1002/art.1780400305.

7. Mishra VV, Goyal PA, Priyankur R, et al. Evaluation of spot urinary albumin-creatinine ratio as screening tool in prediction of preeclampsia in early pregnancy. J Obstet Gynaecol India 2017;67(6):405408. DOI: 10.1007/s13224-016-0950-7.

8. Jasin HE. Autoantibody specificities of immune complexes sequestered in articular cartilage of patients with rheumatoid arthritis and osteoarthritis. Arthritis Rheum 1985;28(3):241-248. DOI: 10.1002/ art.1780280302.

9. Yao Y, Wang SX, Zhang YK, et al. Acquired Fanconi syndrome with proximal tubular cytoplasmic fibrillary inclusions of $\lambda$ light chain restriction. Intern Med 2014;53(2):121-124. DOI: 10.2169/ internalmedicine.53.0836.

10. Raimbourg Q, Daugas É. Lupus nephritis. Nephrol Ther 2019;15(3):174189. DOI: 10.1016/j.nephro.2018.11.003.

11. Farnoodian M, Sorenson CM, Sheibani N. PEDF expression affects the oxidative and inflammatory state of choroidal endothelial cells. Am J Physiol Cell Physiol 2018;314(4):C456-C472. DOI: 10.1152/ ajpcell.00259.2017.

12. Li R, Dai J, Kang H. The construction of a panel of serum amino acids for the identification of early chronic kidney disease patients. Clin Lab Anal 2018;32(3):e22282. DOI: 10.1002/jcla.22282.

13. Martinez-Martinez MU, Sturbaum AK, Alcocer-Varela J, et al. Factors associated with mortality and infections in patients with systemic lupus erythematosus with diffuse alveolar hemorrhage. Rheumatol 2014;41(8):1656-1661. DOI: 10.3899/jrheum.130927.

14. Roelofsen-de Beer RJAC, van Zelst BD, Vroling AB, et al. Ramakers when results matter: reliable creatinine concentrations in hyperbilirubinemia patients. C Clin Chem Lab Med 2019;57(5):659667. DOI: $10.1515 / \mathrm{cclm}-2018-0959$. 